\title{
Latitude, light, clocks and mood
}

\author{
Thomas C Erren • Melissa S Nise • \\ V Benno Meyer-Rochow
}

Received: 14 January 2011 / Accepted: 21 January 2011 / Published online: 19 February 2011

(C) The Author(s) 2011. This article is published with open access at Springerlink.com

"Illumination and mood" (Kripke 2011) has been the common theme of three recent contributions to the journal. In August 2010, Dr. Hoaki and colleagues reported that, among 56 healthy volunteers, higher illuminances during the day and greater variability in sleep times predicted hyperthermic temperament scores (Hoaki et al. 2011). With regard to "light", Dr. Kripke asked whether the converse applied to the sample as well, namely that low illumination and depression were linked (Kripke 2011). In October 2010, Terao and Hoaki concluded from additional analyses "that light can ameliorate low mood in healthy people" and closed with the suggestion that "further studies are required to prospectively investigate whether light can prevent the occurrence of depression in healthy people" (Terao and Hoaki 2011).

Such-and more related-studies with regard to the critical role of light for "hyperthermic temperament", "hypomania or mania" and "depression" to which Dr. Kripke points, may benefit from recent results by Ciarleglio et al. (2011). Indeed, brought together, the aforementioned publications may form the basis for refined tests of two related hypotheses, namely (i) the instability hypothesis of bipolar disorder (Goodwin and Jamison 1990) and (ii) the latitude hypothesis of an association between latitude and seasonal affective disorders (SAD) (Potkin et al. 1986).

\footnotetext{
T. C. Erren $(\bowtie) \cdot$ M. S. Nise

Institute and Policlinic for Occupational Medicine, Environmental

Medicine and Prevention Research, University of Cologne,

Cologne, Germany

e-mail: tim.erren@uni-koeln.de

V. B. Meyer-Rochow

School of Engineering and Science, Jacobs University Bremen, 28759 Bremen, Germany
}

Here is why and how: Ciarleglio et al. (2011) reported that perinatal light exposures imprint circadian clocks with lasting effects on the alignment of clock rhythms to light and on the circadian behaviour in animals. Remarkably, when mice were raised under "winter light conditions", a key developmental consequence was an inappropriate entrainment of their biological clocks and rhythms to changing seasonal photoperiods later in life. In fact, mice raised under 'winter light conditions' evinced a large variability in the timing of their master clocks relative to seasonal light/dark transitions. Interestingly, human SAD sufferers exhibit enhanced circadian responses to seasonal light cycles which are similar to the ones detected in the short-day matured mice (Ciarleglio et al. 2011; Wehr et al. 2001). While these experimental findings must, clearly, be replicated and substantiated beyond the observed weeks, they could, equally clearly, open doors to better understand facets of the postulated biologically plausible nexus of 'light and mood', in general, and of hypotheses (i) and (ii), in particular.

Regarding hypothesis (i), the study results by Dr. Hoaki and colleagues could now be followed up insofar as further investigations should consider season and location of birth of the study participants. Indeed, including the variables of when and where study individuals were born may ultimately explain why, among study individuals who share the same exposures to light, some may show more pronounced indications of hyperthymic temperament scores, or depression, or why some develop these conditions earlier and others later. In addition, it seems reasonable to consider when and where the study participants were born in studies which contrast groups with assumed major differences in their average illuminances as well. In such studies, the time of year and location of an individual's birth could be covariates which might help to explain within-group variations of 
hyperthermic temperament or "depression" (Kripke 2011) in future studies.

Regarding hypothesis (ii), it certainly is worth following up whether when and where (i.e. season and geographic location) people are born could make them susceptible to mood disorders later in life. In human populations, compared with the situation newborns face at lower latitudes, those born at extreme latitudes, where the time window for "winter light conditions" is larger and the light/darkness quotient smaller, should experience stronger signals capable of imprinting circadian clocks. As one result, at extreme latitudes, more babies can be expected to be born during periods of extreme light/dark ratios, i.e. winter seasons, than, for instance, at the equator. As a further result, those born in the extreme northern, or southern, regions of the world shortly before or during extended winter months could be expected to have increased risks of developing neurobehavioral disorders, such as SAD, later in life. Studies into the possible effects of light on seasonal affective disorders, utilizing extreme light/dark ratios in people inhabiting polar regions, have been suggested earlier, for instance, in 2007 (Erren et al. 2007). But given recent ambiguous results on the postulated causal link between latitude and SAD (Brancaleoni et al. 2009; Kegel et al. 2009), one should now be in a position to conduct targeted experiments that support and direct epidemiological studies into the aetiology of the disease. Importantly, future observational studies could soon zero in on two questions. First, are SAD rates in cohorts of individuals born in winter months at extreme latitudes higher than in cohorts who are born at other times of the year there and/or in locations closer to the equator? Second, is the likelihood of having been born in winter months and at extreme latitudes higher in cases with SAD than in controls without the disease?

It is worth noting that epidemiologists, unaware of biologically plausible mechanisms, often have to embark on "black-box" or "stabs in the dark" epidemiology which can render research ineffective. The interesting findings reported in Psychopharmacology and Nature Neuroscience since August 2010 may now provide the basis for "whitebox" epidemiology (Erren 2010), in which observational studies into the two guiding hypotheses could be targeted via the reported insights. Importantly, please note that, beyond studies which may be planned now, in neurobehavioral disorder studies which are already completed, there may be value to conduct additional analyses that consider the time and place of birth as variables a posteriori, i.e. today in the light of the recent study findings.
Overall, the results reported by both Hoaki et al. (2011) and Terao and Hoaki (2011) and the findings reported by Ciareglio et al. (2011) are compatible with the notion that instable, rather than robust, circadian systems may make humans susceptible to several "mood disorders", including bipolar and seasonal affective disorders. The hypotheses (i) and (ii) are then integral parts of this scenario. Remarkably, when and where people are born might be an indicator or predictor of a critical vulnerability of the circadian system which may predispose to the onset, and shape the course, of adverse effects on mood.

Acknowledgements TCE would like to thank the UC Berkeley's School of Public Health for having provided a stimulating environment during his work as a visiting scholar between July 2010 and January 2011 .

Open Access This article is distributed under the terms of the Creative Commons Attribution Noncommercial License which permits any noncommercial use, distribution, and reproduction in any medium, provided the original author(s) and source are credited.

\section{References}

Brancaleoni G, Nikitenkova E, Grassi L, Hansen V (2009) Seasonal affective disorder and latitude of living. Epidemiol Psichiatr Soc $18: 336-343$

Ciarleglio CM, Axley JC, Strauss BR, Gamble KL, McMahon DGA (2011) Perinatal photoperiod imprints the circadian clock. Nat Neurosci 14:25-27

Erren TC (2010) Shift work, cancer and "white-box" epidemiology: association and causation. Epidemiol Perspect Innovat 7:11-18

Erren TC, Meyer-Rochow VB, Erren M (2007) Health clues from polar regions. Science 316:540

Goodwin FK, Jamison KR (1990) Sleep and biological rhythms. In: Manic-depressive illness, 1st edn. Oxford Univ. Press, New York, pp 541-574

Hoaki N, Terao T, Wang Y, Goto S, Tsuchiyama K, Iwata N (2011) Biological aspect of hyperthymic temperament: light, sleep, and serotonin. Psychopharmacology (Berl) 213:633-638

Kegel M, Dam H, Ali F, Bjerregaard P (2009) The prevalence of seasonal affective disorder (SAD) in Greenland is related to latitude. Nord J Psychiatry 63:331-335

Kripke D (2011) Illumination and mood. Psychopharmacology (Berl) 213:833

Potkin SG, Zetin M, Stamenkovic V, Kripke D, Bunney WE Jr (1986) Seasonal affective disorder: prevalence varies with latitude and climate. Clin Neuropharmacol 9(Suppl 4):181-183

Terao T, Hoaki N (2011) Light can ameliorate low mood in healthy people. Psychopharmacology (Berl) 213:831

Wehr TA, Duncan WC Jr, Sher L, Aeschbach D, Schwartz PJ, Turner EH, Postolache TT, Rosenthal NE (2001) A circadian signal of change of season in patients with seasonal affective disorder. Arch Gen Psychiatry 58:1108-1114 